\title{
Nailfold vascular findings of anti-melanoma differentiation-associated gene 5 antibody- positive patients with dermatomyositis
}

\section{Kazuhiro Komura: Dermatology, Kanazawa Hospital, Kanazawa, Japan and Dept of Dermatology Municipal Tsuruga}

Hospital

\section{Minoru Hasegawa: Dept of Dermatology, University of Fukui, Fukui, Japan}

Background and aims. Rapidly progressive interstitial lung disease is complicated in the majority of patients with dermatomyositis who are positive for anti-melanoma differentiation- associated gene 5 (MDA5) antibody. Clinically amyopathic dermatomyositis with heliotrope rash and Gottron's sign can offer an implication for anti-MDA5 antibody positivity and the prompt treatment before uncovering the results of blood testing, since these patients can survive only if they received immediate and intensive therapy. We observed nailfold capillary formation of two dermatomyositis patients with anti-MDA5 antibody using dermoscopy in the current study in order to test the capability to estimate the anti-MDA5 antibody positivity.

Methods Nailfold of two patients with dermatomyositis were examined using dermoscopy (DermLite 3, 3Gen). MDA5 positivity was confirmed by ELISA. The images were compared to the dermatomyositis patient with antibodies against Jo-1 or TIF1, and systemic sclerosis patients with anticentromere antibody.

Results Marked haemorrhages and enlarged capillaries were observed in almost all nailfolds of both hands in patients with ant-MDA5 antibody, while only up to three nailfolds in patients with antibodies against Jo-1, TIF1, or centromere. Loss of capillaries was not detected in patients with anti-MDA5 antibody under the dermoscopy at all, although it has been reported in the literature that loss of capillaries can be detected by capillaroscopy.

Conclusions The results of the current study suggest that nailfold findings using dermoscopy have a potential to diagnose the patients anti-MDA5 antibody at their first visit, although there is a limitation in the number of patient samples in this study. Likewise, the nailfold findings on dermoscopy may provide visible information for the

pathogenesis of interstitial pneumonitis in these patients as well.

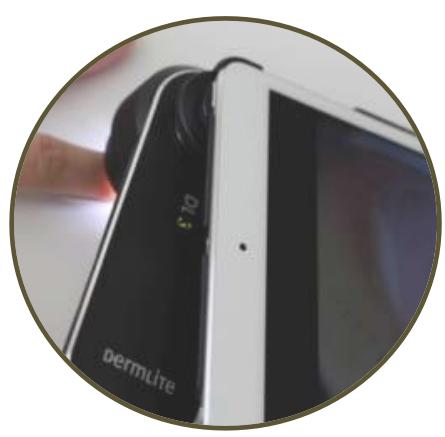

Comparison between patients w/auto-Abs
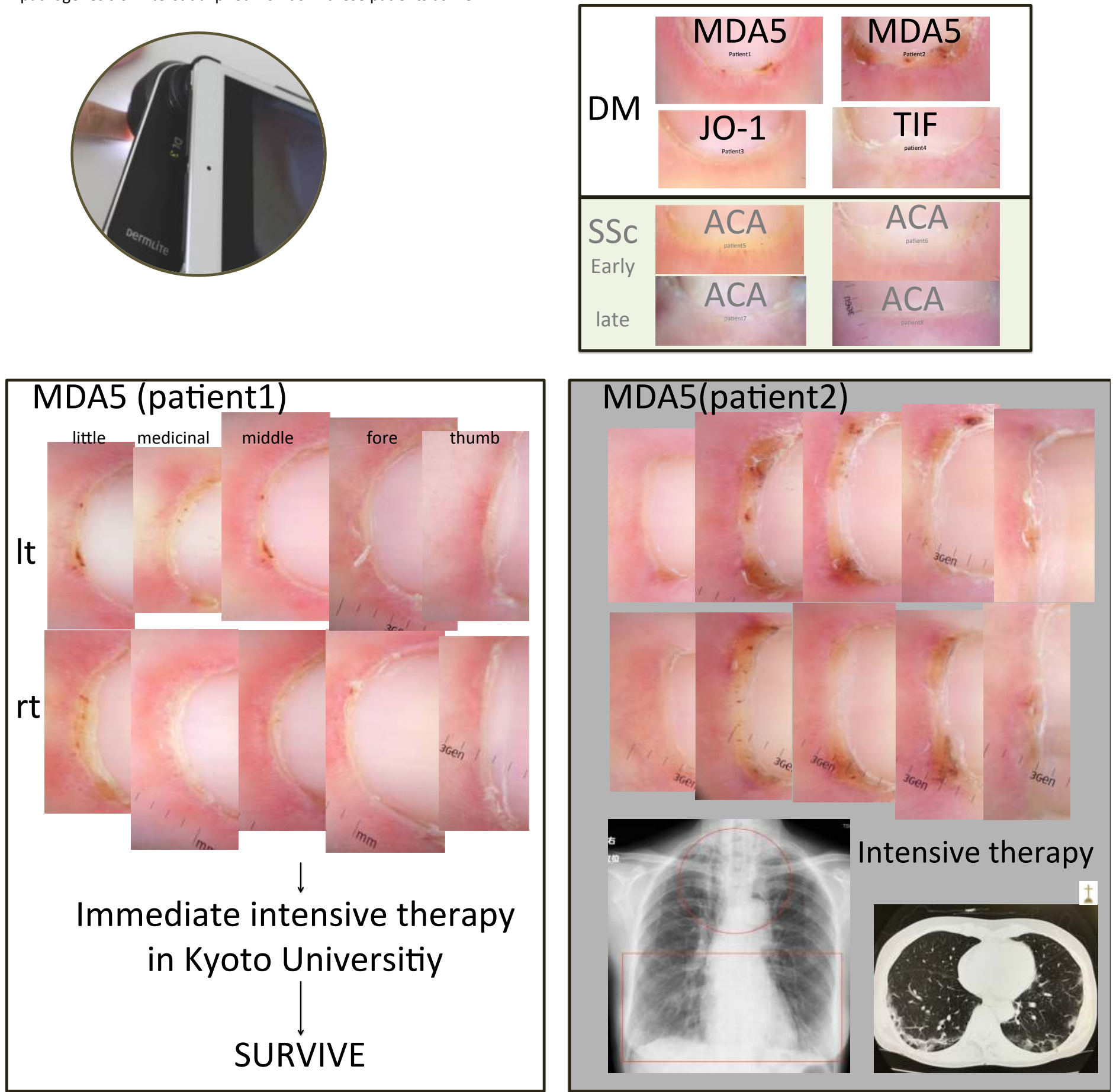